

\title{
PLACEMAKING - TRANSFORMACIÓN DE UN LUGAR EN EL ASENTAMIENTO HUMANO SANTA JULIA, PIURA, PERÚ
}

\author{
PLACEMAKING: THE TRANSFORMATION OF A PLACE IN THE SANTA \\ JULIA HUMAN SETTLEMENT, PIURA, PERU
}

\author{
STELLA SCHROEDER \\ Maestria en Urbanismo \\ Coordinadora y Docente Urbanismo en la Universidad de Piura \\ Piura, Perú \\ https://orcid.org/0000-0001-8591-2719 \\ stella.schroeder@gmx.de
}

\author{
CLAUDIA COELLO-TORRES \\ Arquitecta \\ Docente Arquitecturas Universidad de Piura \\ Piura, Perú \\ https://orcid.org/0000-0002-0873-1313 \\ claudia.coello@udep.pe
}

\section{RESUMEN}

Al gestionar los actuales desafíos en el desarrollo urbano, se debe encontrar un equilibrio entre los enfoques de la planificación oficial y las necesidades locales. Las dificultades que enfrenta el desarrollo urbano participativo deben contrastarse con el fenómeno de "hacer ciudades", que se observa en ciudades de América Latina desde hace muchos años. El artículo describe una experiencia en búsqueda a una nueva metodología de hacer intervención en la ciudad y el espacio público que, desde la academia, permitan empoderar a sus habitantes para la reivindicación de sus derechos.

Dicha experiencia consistió en intervenir en un espacio público seleccionado de la ciudad de Piura, Perú. Bajo el término de Placemaking, se articularon objetivos académicos y sociales mediante el mejoramiento de las condiciones físicas de la ciudad. De ella se expone el proceso de trabajo en campo, que permitió identificar las necesidades, afectaciones y vocación del lugar; el cual produjo un diagnóstico que sirvió de base para el desarrollo de los diseños, programar actividades con la comunidad y grupos de apoyo; así como, la gestión de recursos para la construcción y el seguimiento del impacto generado en el lugar.

Palabras clave

espacio público, intervenciones urbanas, asentamientos humanos, utopías

\section{ABSTRACT}

When managing current challenges in urban development, a balance must be found between official planning approaches and local needs. The difficulties faced by participatory urban development should match the phenomenon of "city-making", which has been observed in Latin American cities for many years. This article describes an experience that sought a new methodology for intervention in cities and public spaces which, starting from academia, empowers inhabitants to insist on their rights. This experience consisted in intervening in a public space in the city of Piura, Peru. Under the concept of placemaking, academic and social objectives come together through the improvement of the physical conditions of cities. The fieldwork presented made it possible to identify the needs, pretenses and uses of the place; in turn, a diagnostic was produced that served as the basis for the development of the designs, programming of activities with the community, and support groups, as well as for the management of construction resources and monitoring of the impact on the place. 


\section{INTRODUCCIÓN}

Actualmente el planeta se está convirtiendo en un mundo más urbano. En 2017, 4,1 mil millones de personas (55\% de la población mundial) vivía en zonas urbanas. Eso es el doble que lo que era veinte años atrás (Banco Mundial, 2018). Las ciudades de América Latina se encuentran, asimismo, en una importante transición. También en 2017, la población urbana llegó aquí al 80.7 \% del total (CELADE - División de Población de la CEPAL, 2017).

Las consecuencias sociales y económicas del desarrollo de la ciudad son complejas. Especialmente cuando las ciudades crecen muy rápido y sin control, aparecen problemas con los servicios básicos de los habitantes. Entonces, ¿cómo satisfacer esta demanda de la ciudad? El factor "calidad de vida" se convierte en el objetivo global, ya que determina la estabilidad política, económica y social de los futuros paisajes urbanos.

La ciudad debe ser para su gente, como declara el "derecho a la ciudad" asociado al pensamiento del sociólogo Henri Lefebvre (1967). La proclama describe la segregación socioeconómica en la ciudad en la década de 1960 y la consecuente reapropiación colectiva, en vista de la discriminación de amplios segmentos de la población.

Aunque hoy no prevalece la misma situación, regresa esta llamada y adquiere un nuevo significado: las necesidades básicas para la vida vuelven a ser cada vez más importantes ante la fragmentación, segregación y desigualdad de las ciudades.

Al mismo tiempo, una ciudad se define por sus espacios públicos. Sin espacio público, la ciudad es inimaginable para nosotros. En efecto, "el espacio público define la calidad de la ciudad, porque indica la calidad de vida de la gente y la calidad de la ciudadanía de sus habitantes." (Borja y Zaida, 2003:16). Las diferentes cualidades sociales y estéticas de sus espacios públicos configuran el carácter de las ciudades, más que sus características topográficas o las peculiaridades de sus tipologías de construcción. Es el lugar donde se desarrollan las expresiones comunitarias necesarias para reconstruir redes sociales y mejorar la calidad de vida en la ciudad. En consecuencia, el espacio público debería dar continuidad a la ciudad y mantener un rol importante en el tejido urbano, ser multifuncional, accesible e inclusivo (ibídem).

En la práctica de planificación se ha dado al espacio público un papel decisivo en el desarrollo urbano en general. Una característica común es la ausencia de un espacio público que facilite la vida en comunidad, lo cual se explica desde su origen ligado a procesos urbanos al margen de las disposiciones urbanísticas. Muchas veces nos encontramos con situaciones de este tipo: plazas y parques descuidados, espacios públicos que no responden a las necesidades de la población o al contexto y que, por lo tanto, son zonas inhabitadas, o son lugares cerrados, exclusivos y no disponibles para el uso total de la población (Low, 2005).

En las últimas décadas ha habido un notable mejoramiento en los niveles de dotación de infraestructura básica y equipamiento urbano, así como en las condiciones de habitabilidad de las ciudades, tanto en la calidad de las viviendas, como en la provisión de los equipamientos urbanos. Sin embargo, es mucho lo que falta por hacer para que existan espacios urbanos en los que prevalezcan condiciones materiales y ambientales adecuadas para todos, y se haga efectivo el "derecho a la ciudad". "Las ciudades son capaces de proveer algo para cada uno solamente porque, y solo en el caso de que así sea, han sido creadas para cada uno" (Jacobs, 1967: 273). Por consiguiente, se necesita un nuevo proceso de diseño, uno más centrado en el contexto y en el proceso que en el producto final (Antonopoulou, Chondros y Koutsari, 2015).

El caso de la ciudad de Piura, Perú, constituye un claro ejemplo de la problemática que enfrenta la mayoría de las ciudades latinoamericanas. El fuerte crecimiento de la población se refleja en numerosos signos de congestión de todos los sistemas urbanos. De acuerdo con la percepción ciudadana sobre calidad de vida, la mayor parte de la población no se encuentra satisfecha con el espacio público disponible en el lugar donde vive (Schroeder). Una gran parte de la ciudad no cuenta con una planificación urbanística previa, debido, en gran medida, a la forma de ocupación del suelo, lo cual cause que los espacios públicos, la infraestructura y los servicios de equipamiento no sean suministrados o previstos de antemano.

El proyecto presentado en este artículo consiste en la construcción de un parque para niños, cuyo objetivo es el de materializar una reivindicación urbana a partir del involucramiento y participación activa de los pobladores del lugar. Se buscó transformar un espacio degradado, caracterizado por la inseguridad, la contaminación visual y ambiental, y convertirlo en un lugar de oportunidad, digno y para el disfrute de todos.

Se trata, en última instancia, de alentar la planificación formal de la ciudad, llevando a cabo un proyecto con un enfoque integrador y participativo para alcanzar una mayor sostenibilidad en el desarrollo urbano.

El parque se ubica en el asentamiento humano Santa Julia de la ciudad de Piura. Santa Julia está caracterizado por poseer un alto índice de delincuencia, criminalidad y micro-comercialización de drogas. Es una zona fragmentada donde el espacio público no se disfruta, sino que más bien se evita. Hacen falta espacios públicos y parques infantiles para la gran cantidad de niños en el sector que creció y sigue creciendo de manera informal. 
Ahora bien, hay que señalar que los mismos vecinos han estimulado el proceso de mejoramiento de dicho parque.

En el proyecto se usa el concepto placemaking como nexo entre la sostenibilidad y la habitabilidad. Este placemaking proporciona acciones y resultados concretos que fomentan objetivos de sostenibilidad más amplios, como el crecimiento inteligente, la accesibilidad a pie y en bicicleta, el transporte público o el espacio urbano pertinente.

\section{MARCO TEÓRICO}

Una ciudad debe ser planificada para la gente que vive en ella y debe ser capaz de responder a las necesidades y deseos de sus habitantes. El arquitecto Jan Gehl critica, en este marco, el descuido de la dimensión humana en la planificación de las ciudades. Tanto las ciudades antiguas como las nuevas requieren redefinir los supuestos en los que se planifican y priorizan los proyectos, centrándose más en las necesidades de las personas (Gehl, 2011). En la práctica actual de muchas ciudades y municipios en todas partes del mundo, los propios habitantes están poco informados sobre los proyectos en el desarrollo urbano. Existe, de esta forma, una falta de integración y participación en la planificación actual.

El término "placemaking" (construir lugares) se entiende como una herramienta para la planificación, diseño y gestión de espacios públicos con un enfoque comunitario. Puede verse como una manera de transformar los no-lugares ("non-lieux") (Augé, 1995). Según Augé, un no-lugar es un lugar que uno ignora o evita, como aparcamientos, terminales de transporte, terrenos baldíos. No son lugares porque carecen carácter y no son atractivos para nadie.

En términos generales, esta perspectiva se refiere a la estrategia de un desarrollo económico de "crear lugares de calidad en los que las personas quieran vivir, trabajar, jugar y aprender" (Wyckoff, 2014:2). La científica de planificación británica Patsy Healey (1997) concibe el placemaking como un enfoque de la planificación territorial, basado en la búsqueda de la mejora de la calidad del espacio y la calidad de vida. La discusión sobre la comprensión del lugarno solo facilita el proceso de planificación, sino también "la idea del lugar tiene un rol para definir la identidad de grupos sociales y esta identidad colectiva debería contribuir a generar cohesión social" (de Magalhaes, Healey y Madanipour, 2002:53). El placemaking puede contribuir así a la formación de la identidad y la creación de cohesión social y, por ende, tener un efecto de estructuración social. Más que simplemente promover un mejor diseño urbano, facilita los patrones creativos de uso, prestando especial atención a las identidades físicas, culturales y sociales que definen un lugar. Se trata de mirar, escuchar y hacer preguntas a las personas que viven, trabajan y juegan en un espacio determinado, para descubrir sus necesidades y aspiraciones (Project for Public Spaces, 2018). En este contexto O'Rourke y Baldwin sugieren que placemaking se considera como un proceso que persigue "involucrar a las personas en la apariencia, la sensación y el funcionamiento de sus espacios públicos para descubrir lo que quieren y esperan de un espacio" (2016:103).

Los inicios del concepto de placemaking se derivan de los trabajos sobre el lugar, donde Relph (1976) y Tuan (1977) establecen una categorización temática y basada en la experiencia, respectivamente, para explicar la "conexión" con un entorno existente. En un sentido de diseño urbano, el proceso (y el concepto) de placemaking, evolucionó a partir de las investigaciones de Whyte (1980) de las plazas públicas y su uso (o falta de ellas) en Nueva York.

Como herramienta, el placemaking ha evolucionado a lo largo del tiempo. Lo que comenzó como un proceso de activismo y protestas contra el desarrollo indomable en ciudades estadounidenses, ahora se ha convertido en un instrumento para unir comunidades y vecindarios que pone énfasis especial en el proceso, y no solo en el resultado final (Silberberg, 2018).

De esta manera, la participación ciudadana juega un rol muy importante. Involucrar a los grupos-objetivo representa una condición indispensable para el éxito y la sostenibilidad de proyectos y programas, y permite a la población local formar parte en las decisiones políticas, sociales y económicas. La trazabilidad de las decisiones aumenta la aceptación y, por lo tanto, la longevidad de los proyectos (Imparato y Ruster, 2003). Vaiou, además destaca que "la participación de este tipo, articulada en torno a las reconfiguraciones del espacio público, apunta a formas de ciudadanía (urbana) y subraya la necesidad de considerar las dinámicas de base expresadas en la participación, junto con su función de formación de espacio" (2018:190).

Esta perspectiva de transformación urbana se preocupa por proyectos bottom-up (de abajo hacia arriba), procesos de empoderamiento y apropiación de los ciudadanos que busca que ellos sean partícipes en la toma de decisiones de las intervenciones en los espacios públicos, considerándolos como los expertos de su entorno más próximo y como actores vitales para la generación de lugares, que faciliten el compromiso cívico y la interacción de la comunidad Webb, 2013; Project for Public Spaces, 2016)

Las actividades muchas veces están lideradas por movimientos de colectivos o vecinos (Spataro, 2016), cuando los ciudadanos son acertados e intervienen en el tejido urbano, los gobiernos pueden llegar a ser considerados incapaces o indeseables. En este contexto, se puede decir que, si bien el placemaking se relaciona con las prácticas formales de planificación urbana, también lo hace con las prácticas informales como, por ejemplo, el urbanismo DIY ("hacerlo tú mismo") (Iveson, 2013), el urbanismo táctico (Lydon y Garcia, 2015) o el urbanismo cotidiano (Chase, Crawford y Kaliski, 1999). El urbanismo informal se caracteriza frecuentemente por prácticas espaciales a menor escala. En esta línea, Finn (2014: 381) señala que estas actividades son "soluciones a menudo innovadoras, sofisticadas y de bajo costo para problemas urbanos difíciles o no abordados". Diferentes grupos, pequeñas empresas, relaciones locales y materialidades, crean sinergia para producir 
intervenciones dinámicas que respondan a las necesidades cambiantes de los pobladores del lugar. No obstante, las interacciones formales e informales de placemaking no son de ninguna manera exclusivas o perfectamente separables. Fabian y Samson (2016), entre otros, argumentan que los planificadores y los diseñadores urbanos han mostrado un interés creciente en el urbanismo DIY.

En conclusión, los diferentes actores producen espacios de su interacción social y contribuyen a dar forma al espacio público, mientras se crea una ciudadanía urbana (Vaiou, 2018). Los beneficios de emplear adecuadamente esta metodología conllevan a la generación de impactos positivos en el entorno urbano, pues un lugar exitoso promueve la comodidad, la salud y el bienestar en las personas, potencia la accesibilidad, fortalece la vida comunitaria y desarrolla la economía local (Karacor, 2014; Antonopoulou, Chondros y Koutsari, 2015; Project for Public Spaces, 2016). De este modo, se configura un espacio público pertinente al lugar y a su gente.

\section{DESARROLLO}

\section{MÉTODOS}

Utilizar la metodología placemaking resulta pertinente y coherente para enfrentar los desafíos actuales, las necesidades y las expectativas de la comunidad piurana de manera que contribuya a construir un espacio público activo, con identidad y cohesión social. La experiencia que aquí se expone constó de distintas etapas progresivas, empezando desde un nivel macro de la ciudad hasta llegar al nivel micro. A partir de los primeros resultados, se desarrolló una visión a largo plazo y un conjunto de estrategias para alcanzarla.

Siguiendo el método de placemaking, se comienza a trabajar en diferentes sitios en la ciudad de Piura para mejorar el espacio público y la calidad de vida para la población. Se debe tener muy cuenta que el proceso no es estrictamente lineal. Sin embargo, como punto de partida, es imprescindible el contacto con la población.

Así, una de las primeras actividades realizadas consistió en definir quiénes participarían, cómo y cuándo. Otro factor importante fue recoger las opiniones de la población local, lo que sirve para entender y analizar las necesidades, falencias y deseos de la comunidad. Seguidamente, se desarrolló la fase de la propuesta, en la cual la gente también participó y trabajó para cumplir con los planes de desarrollo del proyecto definidos. Además, se realizó un plan de ejecución por etapas el cual contempla que, una vez construido el proyecto, se debe continuar trabajando con la evaluación y el seguimiento del mismo. Conservar estas líneas de continuidad permite la flexibilidad del proyecto para lograr mejoras a lo largo del tiempo, según los objetivos establecidos.
En el transcurso del trabajo en campo se han desarrollado diversas actividades y se ha trabajado de forma permanente con la comunidad del asentamiento humano Santa Julia.

Este asentamiento humano, pertenece al distrito Veintiséis de Octubre y está ubicado al extremo sur oeste de la ciudad de Piura, Perú (figura 1). La evolución de este sector se ha venido dando en tres etapas, las cuales se han establecido por las siguientes modalidades: ocupación informal, construcción, planificación y servicio. Dado su proceso de conformación, la zona no cuenta con actividades económicas promotoras del empleo, las actividades económicas que se registran, son de autoempleo y de pequeños negocios con bajos niveles de permanencia.

Piura a pesar de que es una de las regiones con mayor aporte al PBI nacional, tiene niveles de pobreza monetaria que alcanzan el 35\%. Ciertamente, la zona de estudio se caracteriza por ser urbano marginal (Municipalidad Distrital Veintiséis de Octubre, 2016).

En términos de población no hay datos exactos. Según el Organismo de Formalización de la Propiedad Informal (COFOPRI) se ha formalizado alrededor de 2,300 lotes. Tomando una aproximación de cinco miembros de familia que ocupa un lote, se suma una población alrededor de 12,000 habitantes. Alrededor del 30\% tienen 14 años o menos y cerca de la mitad tiene menos de 25 años de edad. Solo menos del 10\%, tienen 60 años y más (Municipalidad Distrital Veintiséis de Octubre, 2016). Según estas cifras, la población que habita la zona es bastante joven con una cantidad de niños por encima del promedio.

En varias visitas y convocatorias se realizó una labor continua con la población local y el equipo de la Junta Vecinal. En un primer taller participativo se trabajó en la búsqueda de respuestas a determinadas necesidades, a través de las siguientes preguntas: ¿Cuál es el uso actual del espacio público? ¿Quién está usando el espacio público? ¿Existen problemas / conflictos? En coordinación con grupos vecinales y la municipalidad se trató, entonces, la factibilidad de diferentes proyectos.

En la siguiente fase, se diseñó una visión de un espacio público de prioridad; el propósito fue que los vecinos definieran el uso, expresaran sus ideas para que el parque cumpliera sus necesidades y deseos. Desde ese enfoque, se programaron diferentes fechas para visitas, incluyendo talleres participativos, la presentación, la discusión de la propuesta y la ejecución del parque. El taller de diseño se realizó mediante la utilización de mapas y una maqueta participativa (Figura 2). Con la maqueta quedaron definido los límites para el diseño del parque y tres zonas de diferentes tamaños para juegos infantiles. A partir de ejemplos y buenas prácticas de otros sitios se discutieron diferentes opciones para el diseño del parque. Después del taller, se elaboró una primera propuesta la cual se mejoró en conjunto con los futuros usuarios.

El predio del área que está prevista como parque tiene una superficie total de $2.100 \mathrm{~m}^{2}$. El terreno limita a una calle que, al 

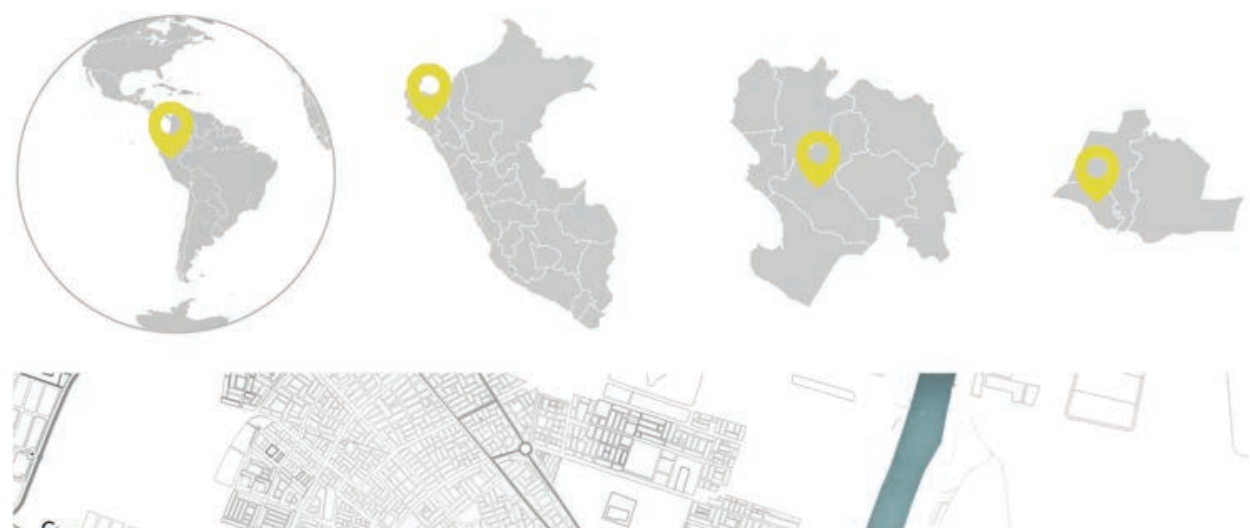

$\sim^{\text {Costa }}$

,
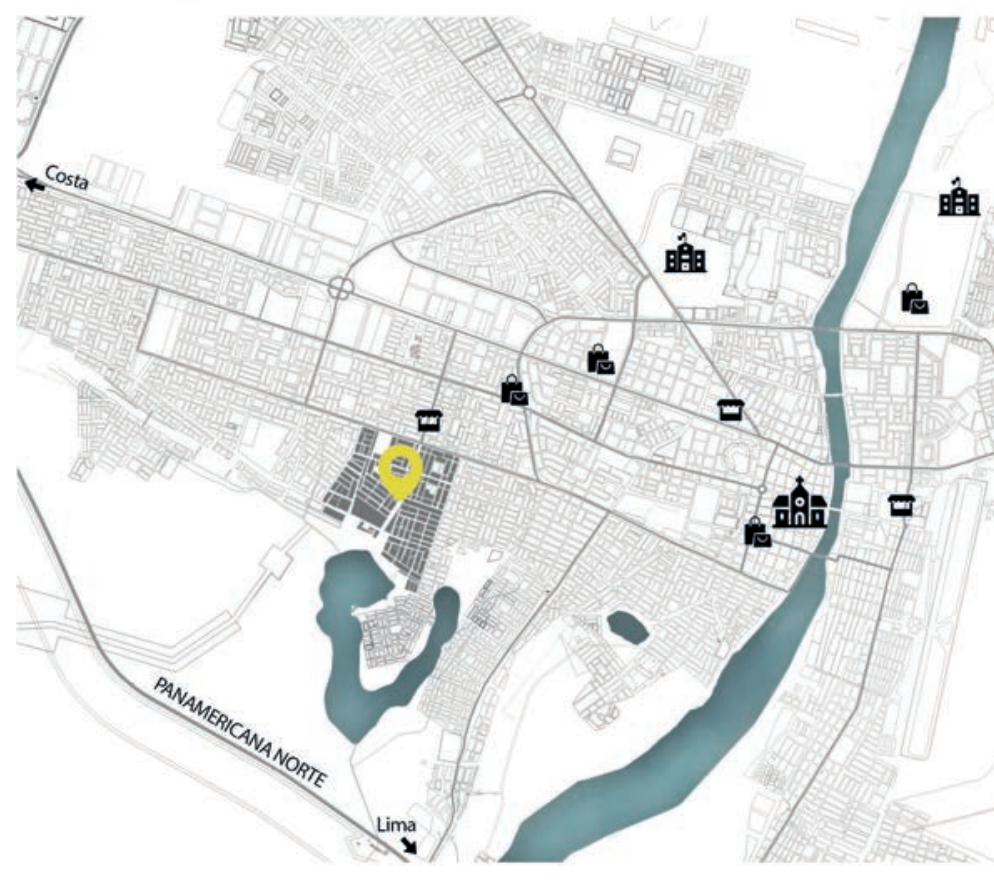

嚕

触 Centrohistorico Pura

解 Universidad

A Centrocomercial

- Mercado

Figura 1. Ubicación del lugar de intervención. Fuente: Elaboración de las autoras.
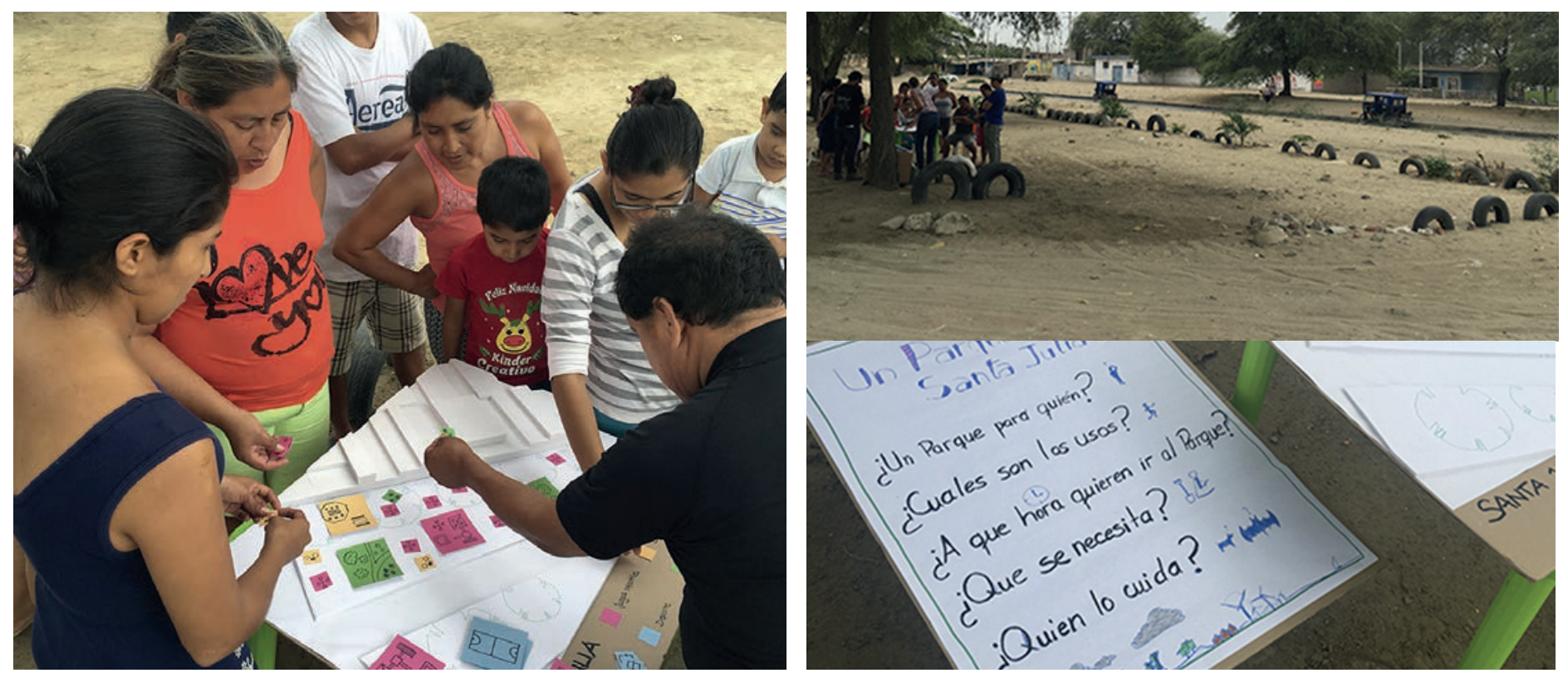


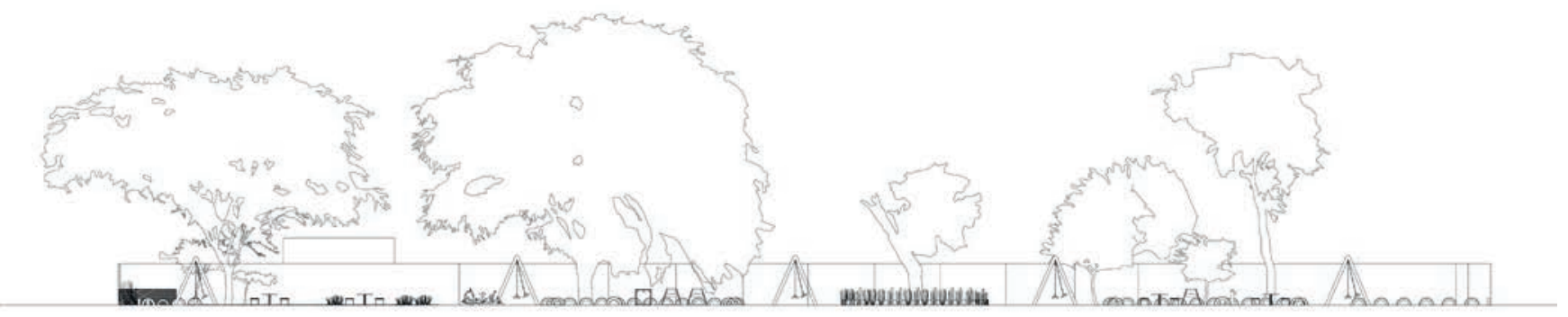

Figura 3. Corte longitudinal del Parque Santa Julia. Fuente: Elaboración de las autoras.

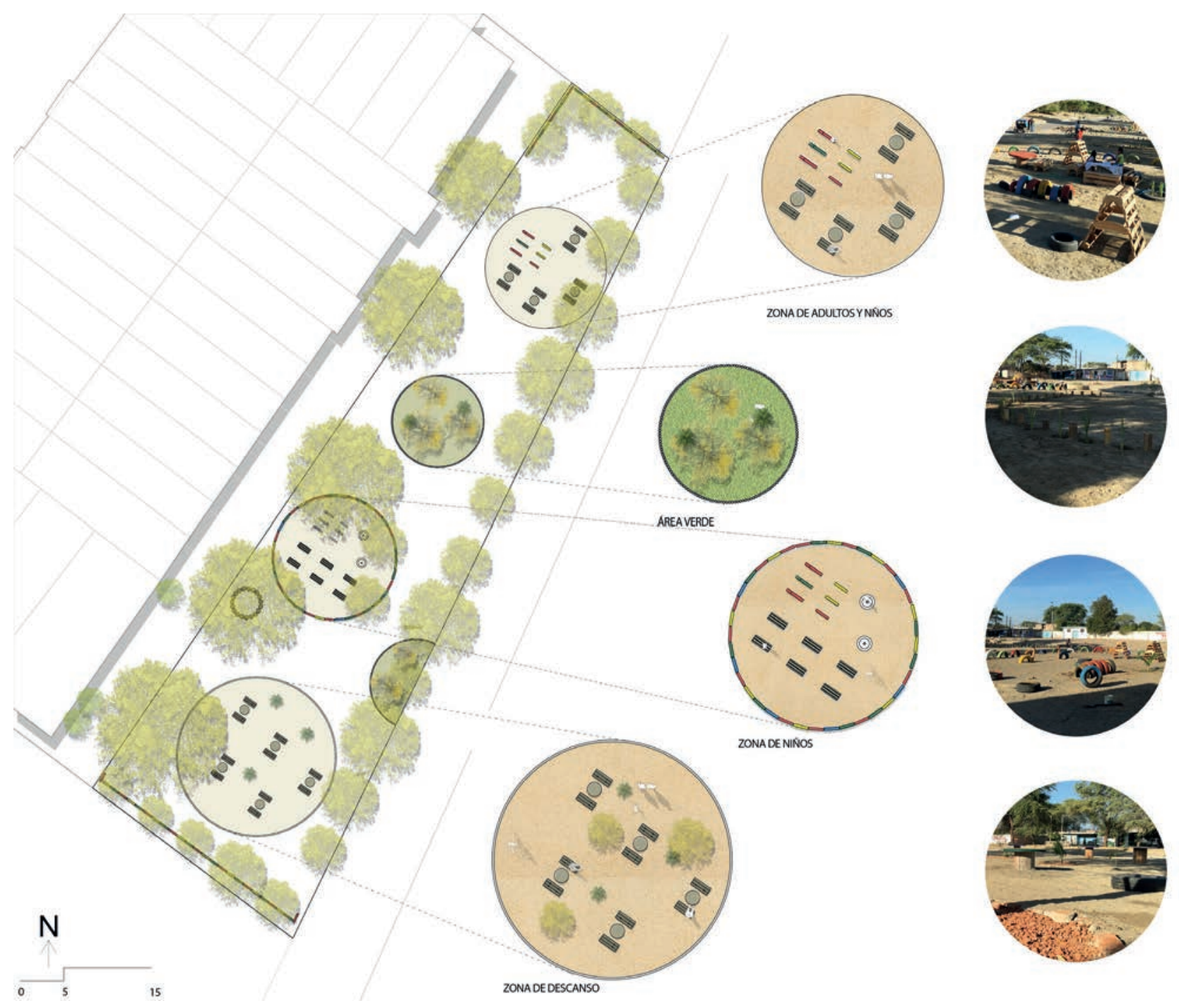

Figura 4. Plano del Parque Santa Julia, realizado a partir de los requerimientos de los vecinos. Fuente: Elaboración de las autoras. 

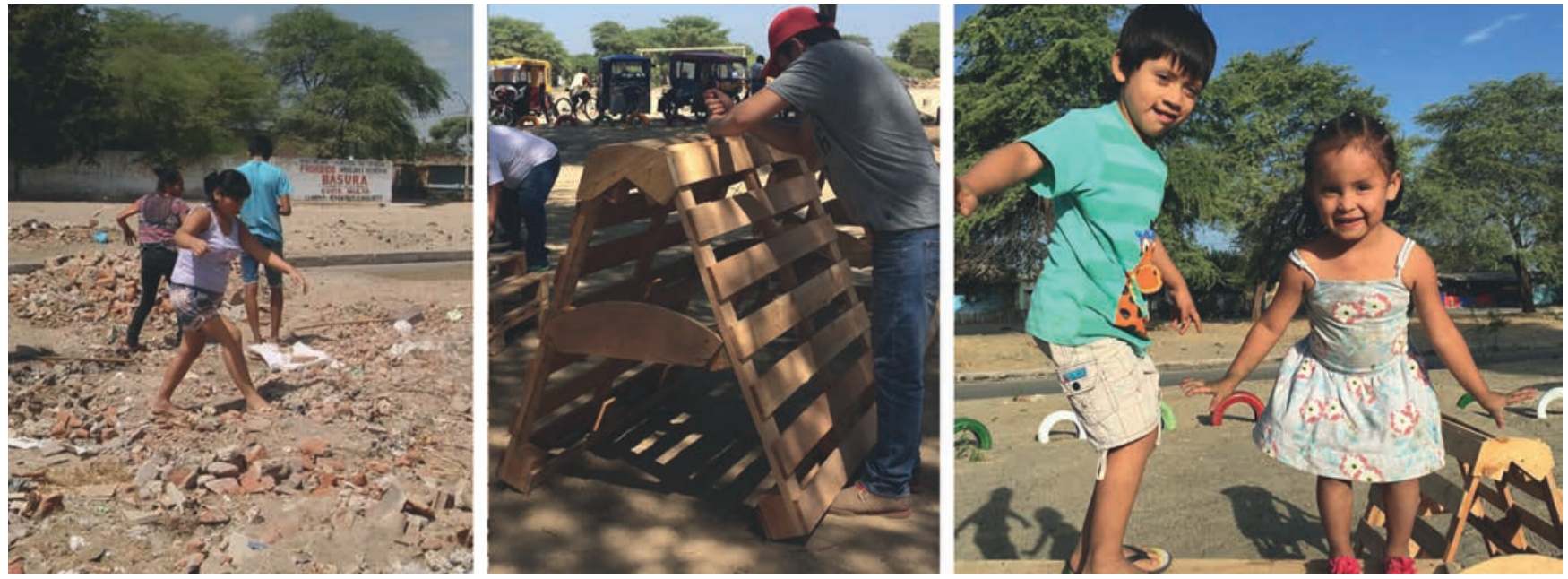

Figura 5. Parte del proceso de ejecución del Parque Santa Julia. Fuente: Fotografías de las autoras.
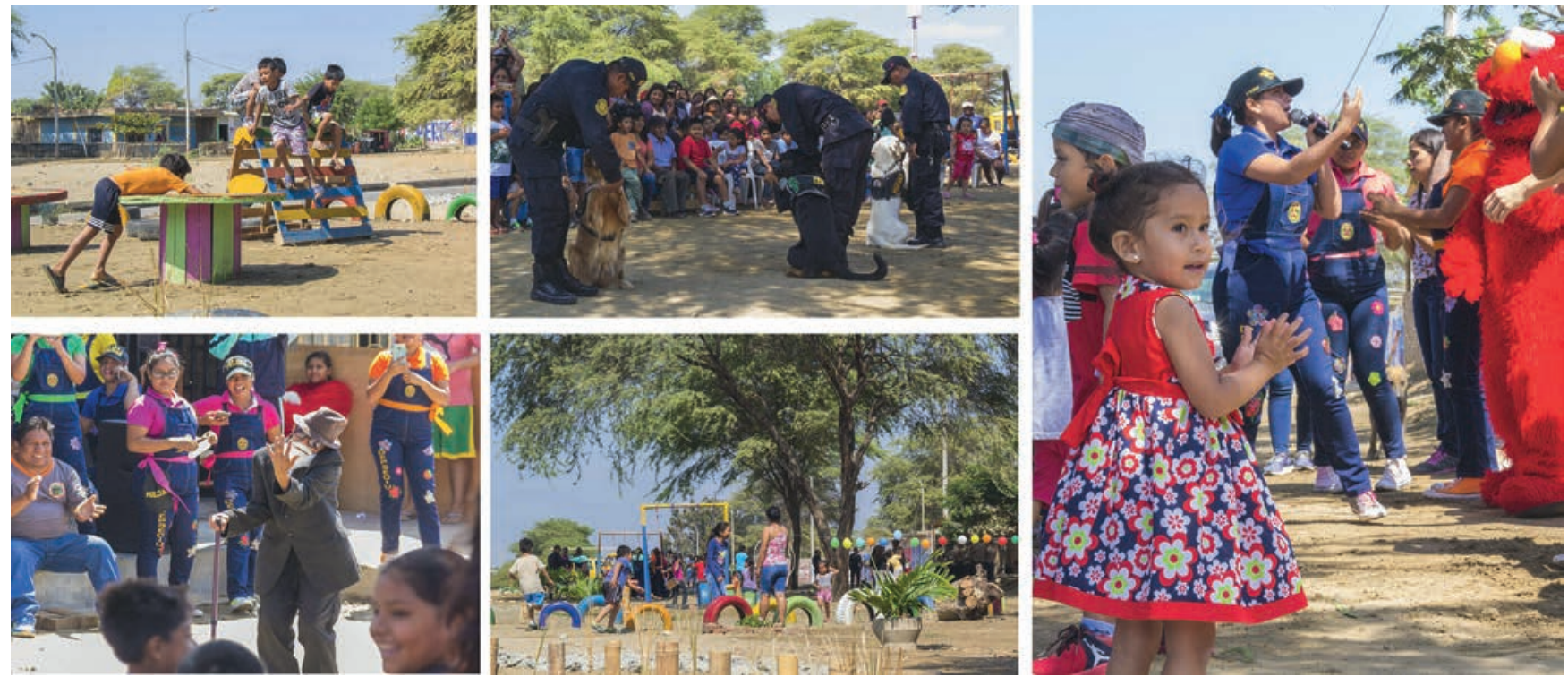

Figura 6. Actividades realizadas el día de la Inauguración. Fuente: Fotografías de las autoras.

mismo tiempo, tiene función de dren y conecta el asentamiento humano con el centro de la ciudad. El costado occidental colinda con viviendas. Todos poseen un acceso directo al mismo. Sobre el terreno, la comunidad tiene el hábito de depositar la basura y los desperdicios de construcción del sector, generando malos olores y contaminación ambiental y visual.

El diseño arquitectónico se ha adaptado a la configuración topográfica actual del terreno. Se ha definido la ejecución de un parque principalmente para niños. Para acompañar las zonas con juegos infantiles, los vecinos establecieron zonas de descanso con bancas y mesas. Las áreas verdes sirven como zonas vacías con una vegetación adecuada al sector (Figura 3 y Figura 4).
Los objetivos para la ejecución del parque estaban relacionados con: el involucramiento de los habitantes en el proceso de toma de decisiones de diseño; el uso de materiales de bajo costo que hicieran factible la replicabilidad futura por los habitantes y la sostenibilidad de la intervención y la planificación de una acción posible de realizar dentro de corto plazo.

La primera actividad de la fase de ejecución, fue una limpieza general del lugar, luego se trabajó en la construcción de los juegos infantiles, del mobiliario y se sembró árboles y plantas. Este procedimiento comprendió 6 semanas de trabajo. En cuanto al criterio ambiental y material, el parque fue construido en gran parte con materiales reciclables y sostenibles como el bambú, palets y llantas en desuso. Los vecinos no solo contribuyeron con ellos sino también con su mano de obra. 

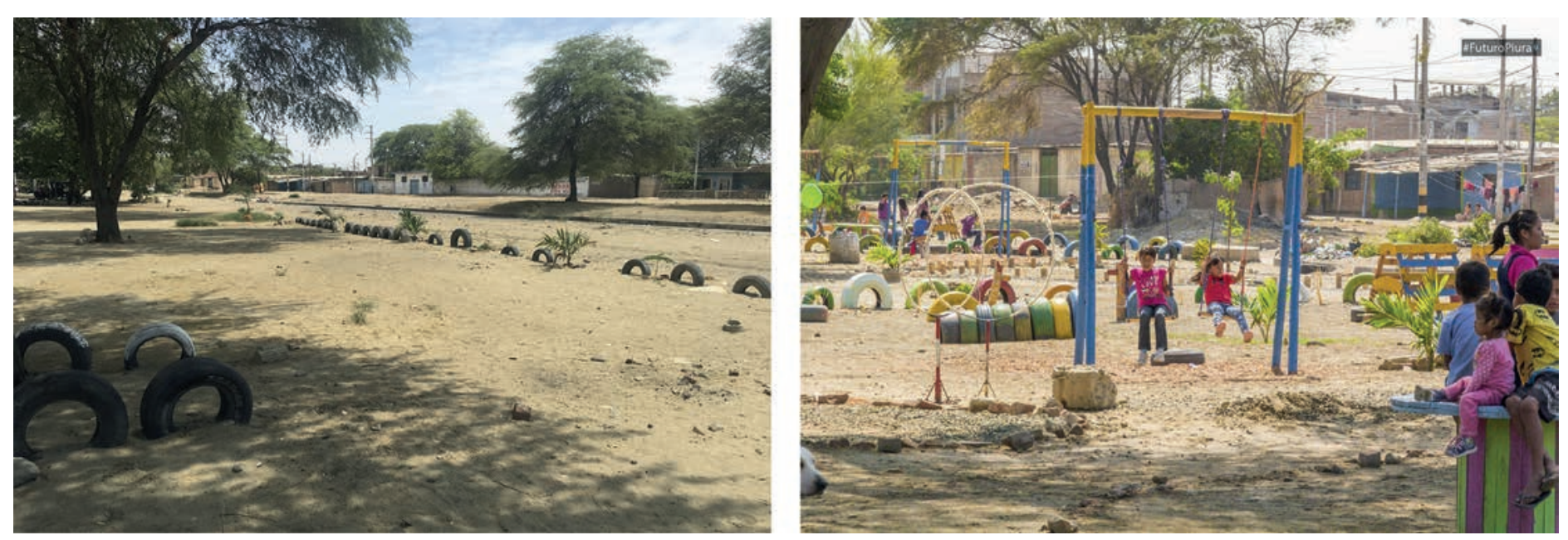

Figura 7. Antes y después. Fuente: Fotografías de las autoras.

Para conseguir los materiales, organizaron varias actividades, como por ejemplo almuerzos colectivos. Algunos materiales como también las plantas fueron donados.

En todos los pasos se trabajó en conjunto con los vecinos y voluntarios (Figura 5). Como proyecto piloto, el parque ha sido inaugurado el 1 de julio de 2018 y debe funcionar como ejemplo para otros asentamientos humanos en la zona (Figura 6).

\section{RESULTADOS}

El trabajo de placemaking continúa mucho después de que se haya instalado el proyecto inicial. Es por eso que la última etapa, consta del seguimiento progresivo, donde se realizan encuestas y diagnósticos con la intención de verificar el grado de aceptación de la propuesta, a partir de las cuales se ejecuten mejoras en el parque y con el objetivo de que se mantenga y aumente el grado de participación comunitaria.

En los trabajos con los vecinos, líderes y representantes de la Junta Vecinal se utilizaron diferentes herramientas, como charlas, redes sociales y talleres para sensibilizar a la población en temas de desarrollo urbano, la importancia del espacio público como también formas sencillas de recuperar los espacios. Las charlas informativas para la población han sido organizadas periódicamente y ofrecieron un espacio para presentar el concepto y la importancia del espacio público. Con las redes sociales, se puso a disposición una plataforma en línea. Se invitó a participar, se convocó a voluntarios y vecinos y se informó sobre los avances del proyecto. En los diferentes talleres participativos se trabajó directamente con la población local para reconocer los deseos y necesidades locales. Se intentó involucrar a todos los vecinos para que se sientan identificados con el proyecto y al mismo tiempo, responsables de su mantenimiento. La Figura 7 muestra las fotografías antes y después de la intervención.

A través de esta intervención, los resultados se manifiestan desde que las personas se interesan por mejorar sus parques, ya que ayudan a crear conciencia a los demás vecinos sobre la importancia de los espacios públicos en la ciudad.

Los vecinos reportan un uso constante del parque, incluso de niños de otros asentamientos humanos y de colegios aledaños. Se logró una mejora de la vida pública en el sector. Las repercusiones han sido favorables e inmediatas, el día de la inauguración del parque, el Jefe de Policías del Equipo de Seguridad Ciudadana del distrito, le entregó un cuaderno de registro al representante de la comunidad, quien se comprometió a mantener una vigilancia diaria en el sector. Además, hubo un acercamiento por parte de la empresa prestadora del servicio público de electricidad, para instalar alumbrado en el parque.

Con el fin de hacer una declaración sobre el impacto de la intervención y la mejora de la calidad y la vida pública en el lugar, se realizó una encuesta con los usuarios del parque cuatro meses después de su inauguración. La recopilación de información se llevó a cabo in situ y ha sido dirigida directamente hacia los usuarios del parque. El diseño del contenido de la encuesta, ha considerado que el espacio cumpla con los criterios que considera Project for Public Spaces ${ }^{1}$ (2015) para que un lugar sea exitoso: el 

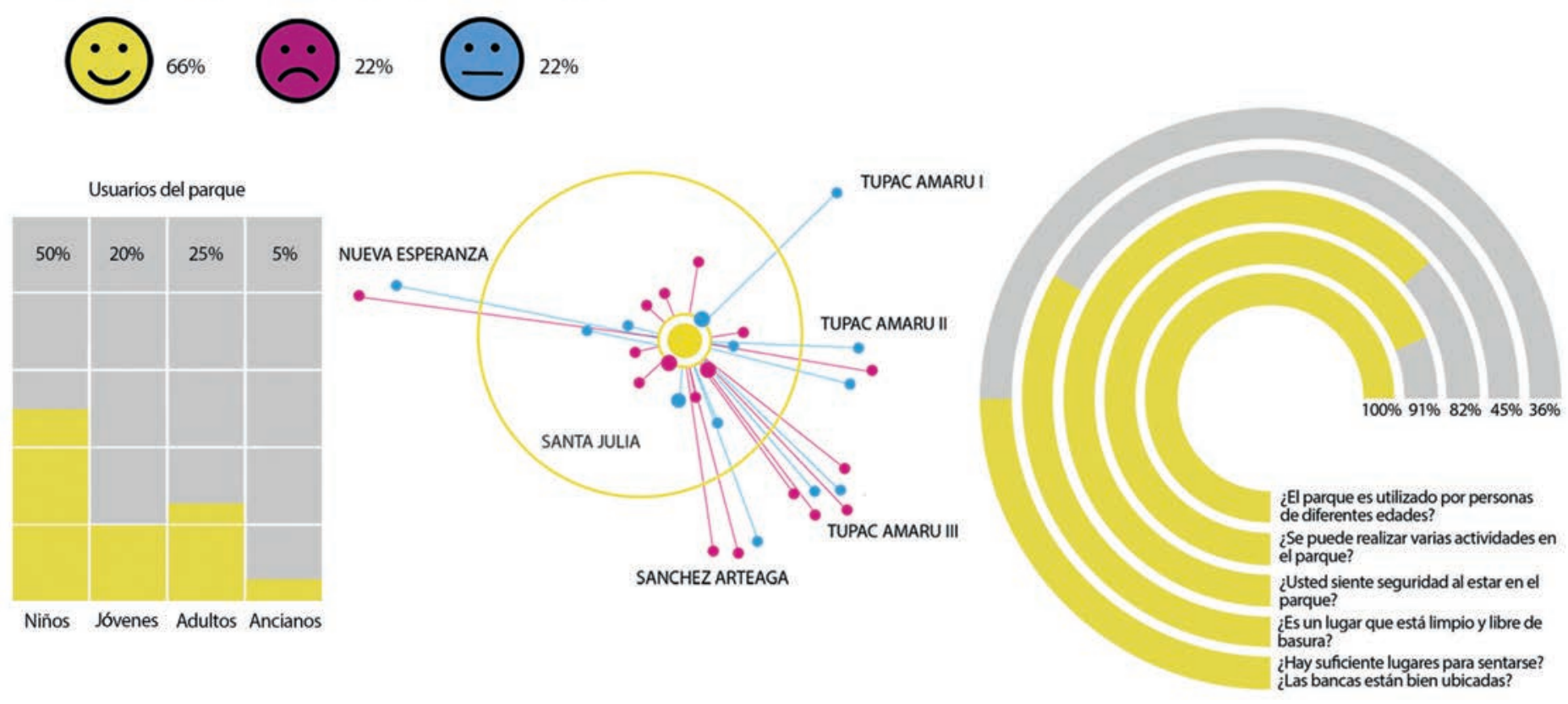

Figura 8. Resultados de encuestas realizada dos meses después de la inauguración. Fuente: Elaboración de las autoras.

encuentro, los usos o actividades, las conexiones, accesos, la comodidad y la imagen. En el caso de evaluar si el lugar actúa como un espacio de encuentro adecuado, se tuvo en cuenta si es un lugar interactivo, si es que existe un uso nocturno, si existe diversidad en los usuarios o si hay un sentido de identidad con el parque. Las preguntas del cuestionario también fueron enfocadas en el dinamismo y uso activo del espacio. La percepción de seguridad y limpieza han sido aspectos importantes en el criterio de comodidad e imagen. Participaron 30 usuarios y vecinos del parque, entre ellos el 40\% fueron niños menores de 12 años.

Los resultados son generalmente positivos (Figura 8). El $66 \%$ de los encuestados consideran que el parque tiene una buena primera impresión. Solo el 22\% no está satisfecho con el impacto visual del lugar. Esta percepción a través de la visión resulta trascendental para contribuir al valor del asentamiento humano y de la ciudad.

Generalmente el parque es utilizado por personas de diferentes edades y la mayoría confirma que el lugar ofrece la realización de varias actividades. El uso constante ha aumentado la seguridad y $82 \%$ de los usuarios entrevistados, dicen sentir dicha seguridad al estar en el parque.

Según los resultados, aún existe un déficit en la limpieza del lugar. Reciclar es un hábito poco incentivado en Piura, además de que la ubicación de los tachos existentes es deficiente, pues se encuentran escondidos de la vista de los usuarios. Asimismo, faltan lugares y bancas para sentarse. Los niños también reclaman mayor variedad de juegos.
Con respecto a evaluar la transformación de un espacio en un lugar vibrante con calidad, que forma una identidad; Project for Public Spaces (2016), ha identificado 11 puntos claves para guiarse. En este sentido, el proceso de la transformación del parque en Santa Julia ha seguido las 11 reglas del placemaking:

1_La comunidad es la experta: Durante todo el proceso se ha trabajado con la población a diferentes niveles de participación.

2_Se crea un lugar, no un diseño: Se ha creado un lugar para niños, donde pueden jugar y pasar su tiempo libre.

3_Es un trabajo en equipo: Los socios han aportado con más recursos, ideas innovadoras y nuevas fuentes de energía: la iniciativa colectiva FuturoPiura, la población, la Junta Vecinal Santa Julia y el Servicio de Seguridad Ciudadana.

4_En el proceso de la ejecución se ha tenido muchas dificultades, pero nunca se ha dicho "No se puede hacer".

5_La mejor manera de cambiar un vecindario es observar lo que funciona y lo que no funciona en ese lugar en particular: Se ha realizado un diagnóstico de la zona y talleres participativos, como mapeos colectivos.

6_Desarrollar una visión: Para que una visión comunitaria haga una diferencia, debe ser creada por las personas que viven allí.

7_La forma soporta la función.

8_Un gran lugar ofrece muchas cosas que hacer: Hay actividades para personas de distintas edades e intereses. 
9_Empezar con pequeñas cosas: Se ha empezado con una estructura básica del parque, que se puede mejorar en el futuro.

10_El dinero no es el problema: Los vecinos han organizado actividades para recolectar fondos. Además, se ha trabajado con materiales económicos, de la zona y/o de reciclaje.

11_Nunca se termina en mejorar un espacio: En conjunto con los vecinos se sigue asegurando el mantenimiento y seguimiento. Se requiere ampliar la oferta de juegos y crear más sombra para los meses de verano. Actualmente, se está buscando materiales para realizar el mejoramiento y se ha implementado una pérgola con la intención de ganar un poco más de sombra.
La Figura 9 muestra los resultados comparativos y actuales del proyecto, utilizando como base los criterios de evaluación del placemaking.

\section{CONCLUSIONES}

Como resultado de un estudio a nivel macro hemos observado que los parques vecinales de Piura tienen problemas para cumplir con su propósito como espacios públicos, debido a la disociación entre las áreas verdes exigidas y construidas y las plazas esperadas. La mayoría de los vecinos pasan su tiempo libre en casa; según ellos, porque no hay lugares públicos adecuados

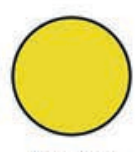

Bueno

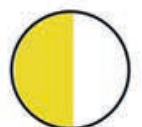

Promedio

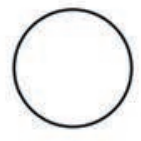

Probre
Antes

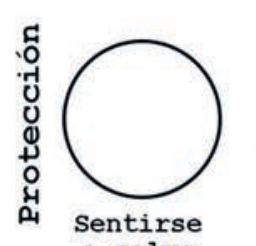

a salvo
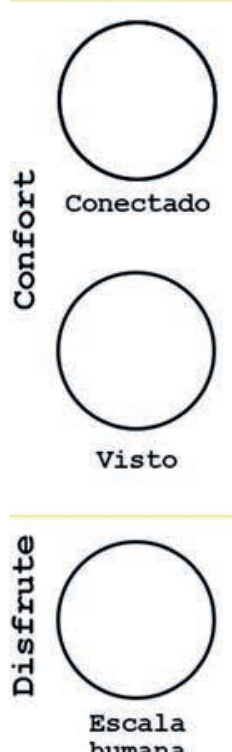
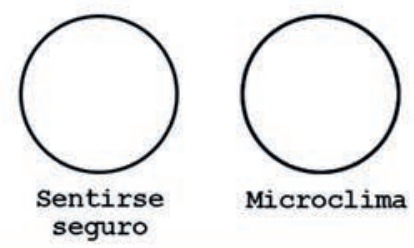

Microclima
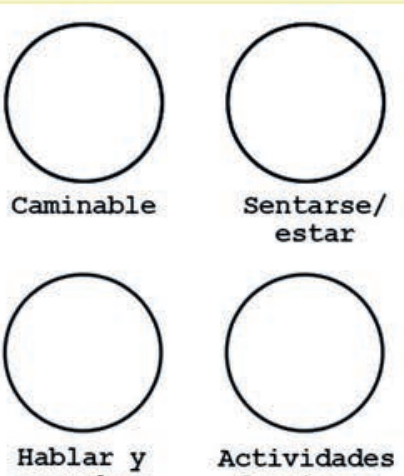
escuchar
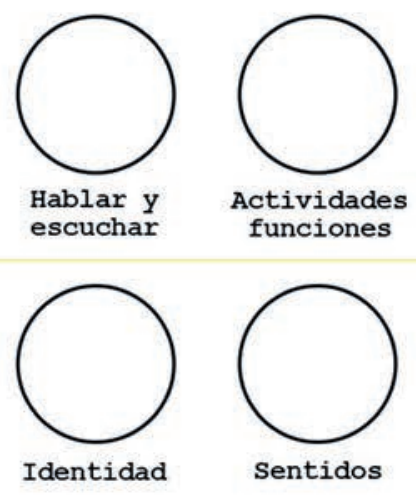

Parque Santa Julia

\section{Después}
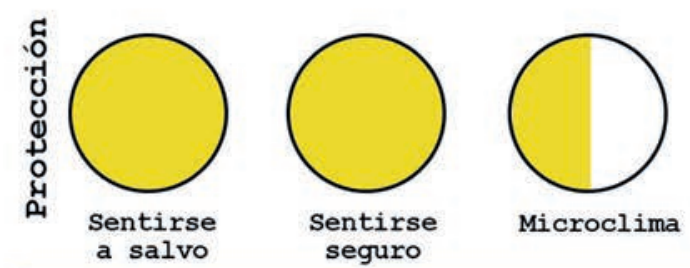
seguro
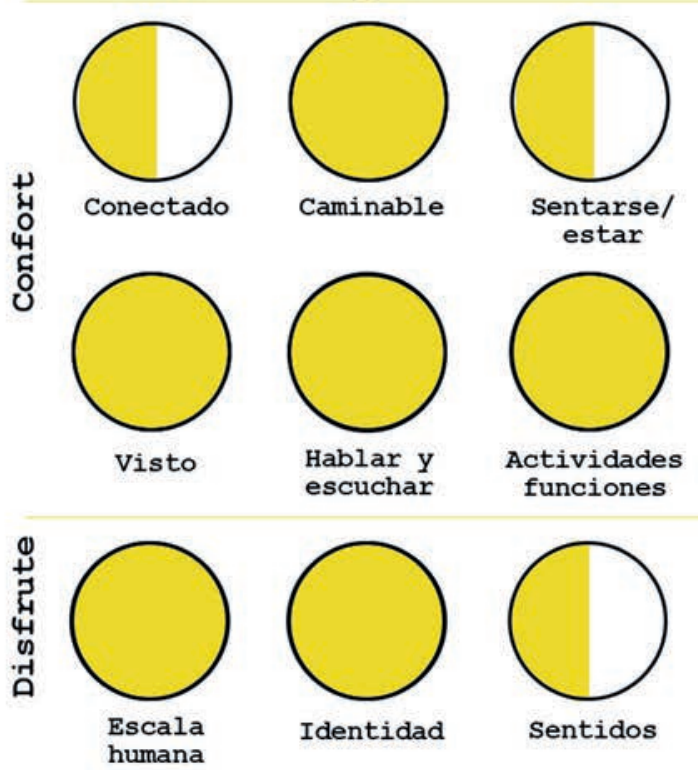
y de calidad; sin embargo, el espacio público expresa las condiciones de la ciudad y juega un rol fundamental y transformador.

Así, a través de talleres participativos se trató de apoyar directamente en el diseño de parques vecinales de Piura. Se intentó lograr una participación en todos los niveles (informar, integrar e incluir) en todo el proceso, identificar usos y por medio del ejercicio de la inclusión, y lograr transformar un contexto específico de manera adecuada.

El proyecto implementó la metodología placemaking porque consideró que la manera más efectiva de mejorar la ciudad es incidir en la preocupación por reinventar los espacios públicos, convirtiéndolos en lugares a partir de los sueños y las necesidades de la comunidad. Siguiendo el pensamiento de Whyte (1980), se puede aprender mucho más observando y hablando con la gente para entender sus necesidades. Además, se tuvo en cuenta que el diseño no es lo más importante, sino un componente para llegar al fin último que es la creación del lugar. De esta forma, en el trabajo concreto se siguió algunos principios básicos que incluyen involucrar a todas las partes interesadas, utilizar métodos visuales y sencillos, evitar la formalización y ser dinámico con los métodos.

El proyecto, desde su perspectiva social, procuró establecer una relación de integración por medio de las diferentes actividades realizadas en el proceso. La intervención, ligada a la regeneración del espacio público, buscó conseguir una red interior que trabajara para establecer una cohesión social mayor, un sentimiento de identificación y responsabilidad y sienta los pilares para una posible conexión al exterior y un diálogo con la ciudad. Se trató de romper las barreras existentes entre la población misma y la ciudad. Al desarrollar una conciencia en las relaciones sociales del espacio público, se pensó en un proyecto dinámico, inacabado y transformable.

La participación de la comunidad ha sido un factor fundamental para el éxito del proyecto cuyo objetivo era alentar la planificación formal de la ciudad y promover el enfoque integrado y participativo para una mayor sostenibilidad, específicamente en el diseño del espacio público, con el enfoque involucrar la población en todas las fases del proyecto. En el espacio público, los vecinos que componen un barrio son los protagonistas del cambio. La participación ciudadana ha operado como eje fundamental; se recopiló el conocimiento de la gente sobre lugares, desafíos y oportunidades. Existe la opinión de que la mejor sostenibilidad de los proyectos se logra con una activa y adecuada participación e integración de la población en todas las etapas de la planificación urbana de su espacio, lo que propició una convivencia colectiva desde antes de la inauguración del parque. En este marco, el placemaking está vinculado a una ciudadanía sostenible. Está impulsado por el proceso, para hacer realidad los lugares y su uso con el fin de promulgar el cambio. El placemaking se caracteriza por ser es un trabajo continuo. Así se asegura la sostenibilidad con diferentes eventos o capacitaciones que se organiza también después de la ejecución del proyecto.

Crear lugares es un trabajo de todos. Los planificadores necesitan involucrar directamente a quienes residen en los vecindarios, y este compromiso significa establecer una relación moral que desde el principio reconoce el derecho de las personas a la ciudad. Este proyecto se diseñó tomando en cuenta las limitaciones con las que cuentan los pobladores y las familias de la periferia de Piura, además de su estrato socio-económico y el impacto social de la imagen del proyecto. Se empleó, cabe mencionar, algunos materiales predominantes de la zona como el bambú, como asimismo materiales reciclados que los propios vecinos pudieron conseguir. En un proceso continuo, los vecinos pueden mejorar su parque de forma autoconstructiva.

Finalmente, el parque ha demostrado un objetivo bastante importante en el debate sobre el espacio público: un lugar para todos.

\section{REFERENCIAS BIBLIOGRÁFICAS}

ANTONOPOULOU, Elene; CHONDROS, Christos y KOUTSARI, Maria. Towards the production of design commons: A matter of scale and reconfiguration. ARQ, 2015, n91, pp. 54-63.

AUGÉ, Marc. Non-Places: Introduction to an Anthropology of Supermodernity. $1^{\circ}$ ed. London, New York: Verso, 1995.

BANCO MUNDIAL. Población urbana / Data [en línea]. 2018. [Consulta: 12 junio 2019]. Disponible en: https:// datos.bancomundial.org/indicador/sp.urb.totl.

BORJA, Jordi y ZAIDA, Muixí. El espacio público, ciudad y ciudadanía. $2^{\circ}$ ed. Barcelona: Electa España, 2003.

CELADE - DIVISIÓN DE POBLACIÓN DE LA CEPAL. Estimaciones y proyecciones de población total, urbana y rural, y económicamente activa. América Latina [en línea]. 2017. [Consultdo 4 junio 2019]. Disponible en: https://www.cepal.org/es/temas/proyeccionesdemograficas/estimaciones-proyecciones-poblaciontotal-urbana-rural-economicamente-activa.

CHASE, John; CRAWFORD, Margaret y KALISKI, John. Everyday urbanism. $1^{\circ}$ ed. New York: Monacelli Press, 1999. 
DE MAGALHAES, Claudia; HEALEY, Patsy y MADANIPOUR, Ali. Assessing institutional capacity for city centre regeneration: Newcastle's Grainger Town. En: CARS, Goran et al. (eds.). Urban Governance, Institutional Capacity and Social Milieux. Aldershot: Ashgate, 2002, pp. 45-69.

FABIAN, Louise y SAMSON, Kristine. Claiming participation - a comparative analysis of DIY urbanism in Denmark. Journal of Urbanism [en línea], 2016, vol. 9, n², pp. 166-184. DOl: https://doi.org/10.1080/175 49175.2015.1056207

FINN, Donovan. DIY urbanism: implications for cities. Journal of Urbanism [en línea], 2014, vol. 7, n 4, pp. 381-398. DOI: https://doi.org/10.1080/17549175.2014 .891149

GEHL, Jan. Life between buildings: Using public space. $6^{\circ}$ ed. Washington: Island Press, 2011.

HEALEY, Patsy. Collaborative planning: shaping places in fragmented societies. $1^{\circ}$ ed. London: Palgrave, 1997.

IMPARATO, Ivo y RUSTER, Jeff. Slum upgrading and Participation. Lessons from Latin America. Washington D.C.: The World Bank, 2003.

IVESON, Kurt. Cities within the City: Do-It-Yourself Urbanism and the Right to the City. International Journal of Urban and Regional Research [en línea], 2013, vol. 37, $\mathrm{n}^{\circ}$ 3, pp. 941-956. DOI: https://doi.org/10.1111/14682427.12053

JACOBS, Jane. Muerte y vida de las grandes ciudades. $2^{\circ}$ ed. Madrid: Capitán Swing Libros, 1967.

KARACOR, Elif. PlaceMaking Approachment to Accomplish Social Sustainability. European Journal of Sustainable Development [en línea], 2014, vol. 3, $\mathrm{n}^{\circ}$ 4, pp. 253-262. http://dx.doi.org/10.14207/ejsd.2014. v3n4p253

LEFEBVRE, Henry. 1967. Le Droit à la ville. L'Homme et la société [en línea], 1967, vol. 6, pp. 29-35. DOl: 10.3917/pal.110.0039.

LOW, Setha. Transformaciones del espacio público en la Ciudad latinoamericana: cambios espaciales y prácticas sociales. Bifurcaciones, 2005, n5, pp. 1-14.

LYDON, Mike y GARCIA, Anthony. Tactical Urbanism: Short-term Action for Long-term Change. $3^{\circ}$ ed. Washington D.C.: Island Press, 2015.
MUNICIPALIDAD DISTRITAL VEINTISÉIS DE OCTUBRE. Plan de desarrollo concertado de distrito de Veintiséis de Octubre 2016-2021 [en línea]. 2016. [Consultado 26 junio 2019]. Disponible en: http://www2.munipiura. gob.pe/institucional/transparencia/pdc-2016-2021.pdf

O'ROURKE, Vicky y BALDWIN, Claudia. Student engagement in placemaking at an Australian university campus. Australian Planner [en línea], 2016, vol. 53, nº 2, pp. 103-116. DOI: http://dx.doi.org/10.1080/072936 82.2015.1135810

PROJECT FOR PUBLIC SPACES. What Makes a Successful Place? [en línea]. 2015. [Consultado 26 junio 2019]. Disponible en: https://www.pps.org/article/ grplacefeat.

PROJECT FOR PUBLIC SPACES. Placemaking: what If we built our cities around places? [en línea]. 2016. [Consultado 26 junio 2019]. Disponible en: http:// www.pps.org/wp-content/uploads/2016/10/Oct-2016placemaking-booklet.pdf.

PROJECT FOR PUBLIC SPACES. How to Turn a Place Around. A Placemaking handbook. $1^{\circ}$ ed. New York: Project for Public Spaces Inc., 2018.

RELPH, Edward. Place and placelessness. $1^{\circ} \mathrm{ed}$. London: Pion, 1976.

SCHROEDER, Stella. Piura al 2032. Una visión urbana de los ciudadanos de Piura para Piura. $1^{\circ}$ ed. Piura: Universidad de Piura, [sin fecha].

SILBERBERG, Susana. Places in the Making: How placemaking builds places and communities [en línea]. Department of Urban Studies and Planning (DUSP), MIT, 2018. [Consultado 26 junio 2019]. Disponible en: http://dusp.mit.edu/cdd/project/placemaking.

SPATARO, David. Against a de-politicized DIY urbanism: Food Not Bombs and the struggle over public space. Journal of Urbanism [en línea], 2016, vol. 9, n² 2, pp. 185-201. DOI: https://doi.org/10.1080/17549175.2015 .1056208

TUAN, Yy-fu. Space and place: the perspective of experience. $8^{\circ}$ ed. Minneapolis: University of Minnesota Press, 1977.

VAIOU, Dina. Rethinking participation: lessons from a municipal market in Athens. Journal of Place Management and Development [en línea], 2018, vol. 11, $\mathrm{n}^{\circ}$ 2, pp. 181-191. DOI: https://doi.org/10.1108/ JPMD-06-2017-0055. 
WEBB, Debra. Placemaking and social equity: Expanding the framework of creative placemaking. Artivate: $A$ Journal of Entrepreneurship in the Arts, 2013, vol. 3, $n^{\circ} 1$, pp. 35-48.

WHYTE, William. The social life of small urban spaces. 6 ed. Washington D.C.: Conservation Foundation, 1980.

WYCKOFF, Mark. Definition of Placemaking: Four Different Types. Planning \& Zoning News, 2014, vol. 32, $n^{\circ} 3$, pp. 1-10. 